\title{
THE MEAN ESCAPE TIME FOR A NARROW ESCAPE PROBLEM WITH MULTIPLE SWITCHING GATES*
}

\author{
HABIB AMMARI ${ }^{\dagger}$, JOSSELIN GARNIER ${ }^{\ddagger}$, HYEONBAE KANG $^{\S}$, HYUNDAE LEE $^{\S}$, AND \\ KNUT SØLNA
}

\begin{abstract}
This paper deals with the narrow escape problem when there are two gates which open alternatively and in a random way. We set up the problem and perform a rigorous asymptotic analysis to derive the mean escape time (MET) for a Brownian particle inside a domain to exit the domain through the switching gates. We show that the leading order term of the asymptotic expansion of the MET is twice the leading order term of the MET when the two gates are open all the time. We also show that the MET decreases as the switching rate between two gates increases. We then consider the case when there are multiple switching gates and derive the leading order term of the asymptotic expansion of the MET.
\end{abstract}

Key words. Mean escape time, first passage, narrow escape problem, time dependent gates, Markov switching, reflecting boundary, Neumann functions, asymptotic expansion.

1. Introduction. Recently the narrow escape problem has attracted a lot of attention in connection with cellular and molecular biology. The narrow escape problem is the problem of computing the mean escape time (MET), or the mean first passage time (MFPT) for a Brownian particle that moves in a microdomain before it exits the domain through a narrow gate on the boundary of the domain. The gate is an absorbing spot on otherwise reflecting boundary. A main concern for this problem is to drive an asymptotic expansion of the MET when the size of the gate tends to 0 . We refer readers to a review paper of Holcman [7] for an overview of the narrow escape problem.

There have been several significant works deriving the leading order and higher order terms of the asymptotic expansions of MET in two and three dimensions [3, 5, $8,9,10,12,14,15,16,17,18,19]$. These work deal with the narrow escape problem when there are one or several gates and these gates are not fluctuating in time, in other words, the gates are open all the time. However there are some cases when the reactivities of the gates fluctuate in time (see [13] and references therein). In fact, [13] deals with the gated narrow escape problem when the diffusing particle stochastically switches between two states and can exit in only one state. It amounts to a single gate switching randomly between the open and closed states.

In this paper we study the narrow escape problem when there are several switching gates. As the first attempt toward this problem, we deal with the case when there are two gates which open alternatively according to a telegraph process. We formulate the problem and rigorously derive an asymptotic expansion for the MET in this case. The asymptotic expansion is expressed in terms of the sizes of the gates. It also depends on the switching rate between the two gates. One of the major interests of this paper

${ }^{*}$ This work was supported by National Institute for Mathematical Sciences of Korea (2010 Thematic Program, TP1003), NRF grants No. 2009-0090250 and 2010-0017532, and NSF grant DMS0709389.

${ }^{\dagger}$ Department of Mathematics and Applications, Ecole Normale Supérieure, 45 Rue d’Ulm, 75005 Paris, France (habib.ammari@ens.fr).

${ }^{\ddagger}$ Laboratoire de Probabilités et Modèles Aléatoires \& Laboratoire Jacques-Louis Lions, Université Paris VII, 2 Place Jussieu, 75251 Paris Cedex 5, France (garnier@math.jussieu.fr).

$\S$ Department of Mathematics, Inha University, Incheon 402-751, Korea (hbkang@inha.ac.kr, hdlee@inha.ac.kr).

『Department of Mathematics, University of California, Irvine, CA 92697 (ksolna@math.uci.edu). 
is to investigate how the MET depends on the switching rate. We then consider the problem when there are more than two gates which open alternatively according to a Markovian dynamics. We formulate the problem in this case and derive the leading order term of the MET. In our case the switching corresponds to a switching of boundary conditions while in [4] for instance the Markov switching corresponds to a switching of the dynamics itself, which for instance has applications in econometric and financial modeling.

The main findings of this paper are twofold. We show that the leading order term of the asymptotic expansion of the MET is twice that of the MET when there two small gates which are open all the time. It means in particular that the switching rate does not affect the leading order MET (this is the case even if there are multiple switching gates). On the other hand, we show that the next term in the asymptotic expansion of the MET decreases as the switching rate increases. In other words, the more rapid the two gates switches, the faster the particle exits the domain.

This paper is organized as follows. In the next section we formulate the escape problem when there are two switching gates. In section 3 , we derive the relevant integral equations using the Neumann function. In section 4, we derive the leading order term and the first order correction of the asymptotic expansion of the MET when there are two switching gates. Section 5 deals with the case when there are more than two switching gates. This paper ends with a brief conclusion.

2. Formulation of the problem. Let $\Omega$ be a smooth, bounded and simply connected domain in $\mathbb{R}^{2}$. We consider the reflected Brownian particle $\left(\boldsymbol{X}_{t}\right)_{t \geq 0}$ confined to $\Omega$ (in fact, it is not the standard Brownian motion, but the one with the generator $\Delta$ instead of $(1 / 2) \Delta)$. The reflected process $\left(\boldsymbol{X}_{t}\right)_{t \geq 0}$ can be rigorously defined as the unique solution to the following equation [11]:

$$
\boldsymbol{X}_{t}=\boldsymbol{X}_{0}+\sqrt{2} \boldsymbol{B}_{t}-\int_{0}^{t} \boldsymbol{n}\left(\boldsymbol{X}_{s}\right) d L_{s}
$$

where $\left(\boldsymbol{B}_{t}\right)_{t \geq 0}$ is a standard two-dimensional Brownian motion, $\boldsymbol{n}$ is the outward normal vector on the boundary $\partial \Omega$, and $\left(L_{t}\right)_{t \geq 0}$ is a continuous nondecreasing process with $L_{0}=0$ which increases only when $\boldsymbol{X}_{t}$ is on the boundary $\partial \Omega$ :

$$
L_{t}=\int_{0}^{t} \mathbf{1}_{\boldsymbol{X}_{s} \in \partial \Omega} d L_{s}
$$

Here we consider the situation that the boundary $\partial \Omega$ is partitioned into three parts, $\partial \Omega_{1}, \partial \Omega_{2}$, and the complementary $\partial \Omega_{c}$. We model an escape problem in which there are two gates at the arcs $\partial \Omega_{1}$ and $\partial \Omega_{2}$ that open alternatively according to a telegraph process $\left(N_{t}\right)_{t \geq 0}$ with parameter $a$ ( $a$ is the rate of switching between the gates). When the particle hits the open gate it escapes rather than being reflected. The telegraph process $\left(N_{t}\right)_{t \geq 0}$ with parameter $a>0$ is a memoryless continuoustime stochastic process that takes on two distinct values, say 1 and 2 [6]. Given that $N_{0}=1$, its random dynamics can be described by

$$
N_{t}= \begin{cases}1 & \text { if } T_{2 j} \leq t<T_{2 j+1} \text { for some } j \\ 2 & \text { if } T_{2 j+1} \leq t<T_{2 j+2} \text { for some } j\end{cases}
$$

where $T_{0}=0, T_{j}=\sum_{l=1}^{j} \tau_{l}$, and $\left(\tau_{l}\right)_{l \geq 1}$ is a sequence of independent and identically distributed exponential random variables with parameter $a>0\left(\mathbb{E}\left[\tau_{l}\right]=a^{-1}\right)$. The 
dynamics is Markovian and the transition probabilities $p(t, j \mid s, k)=\mathbb{P}\left(N_{t}=j \mid N_{s}=k\right)$ satisfy the master equations:

$$
\begin{aligned}
& \frac{\partial p(t, 1 \mid s, k)}{\partial t}=-a p(t, 1 \mid s, k)+a p(t, 2 \mid s, k), \quad t \geq s, \quad p(s, 1 \mid s, k)=\delta_{1 k}, \\
& \frac{\partial p(t, 2 \mid s, k)}{\partial t}=a p(t, 1 \mid s, k)-a p(t, 2 \mid s, k), \quad t \geq s, \quad p(s, 2 \mid s, k)=\delta_{2 k} .
\end{aligned}
$$

Note that $\left(N_{t}\right)_{t \geq 0}$ is reversible and that its stationary distribution is the uniform distribution over $\{1,2\}$.

The goal is to compute the expectation of the hitting time for the process $\left(\boldsymbol{X}_{t}\right)_{t \geq 0}$ to the time-dependent boundary segment $\partial \Omega_{N_{t}}$ (i.e., $\partial \Omega_{1}$ when $N_{t}=1$ and $\partial \Omega_{2}$ when $N_{t}=2$ ). We denote by $\mathcal{T}$ the stopping time which corresponds to the "escape" of the particle:

$$
\mathcal{T}=\mathcal{T}_{1} \wedge \mathcal{T}_{2}, \quad \mathcal{T}_{j}=\inf \left\{t \geq 0, N_{t}=j \text { and } \boldsymbol{X}_{t} \in \partial \Omega_{j}\right\}, \quad j=1,2 .
$$

The MET is the expectation $\mathbb{E}_{k, x}[\mathcal{T}]$ of the stopping time when the initial states are $N_{0}=k$ and $\boldsymbol{X}_{0}=\boldsymbol{x}$, with $k \in\{1,2\}$ and $\boldsymbol{x} \in \Omega$. If $N_{0}$ follows the uniform distribution over $\{1,2\}$, then the MET is given by $\mathbb{E}_{u, x}[\mathcal{T}]$ where

$$
\mathbb{E}_{u, \boldsymbol{x}}[\mathcal{T}]=\frac{1}{2}\left(\mathbb{E}_{1, \boldsymbol{x}}[\mathcal{T}]+\mathbb{E}_{2, \boldsymbol{x}}[\mathcal{T}]\right)
$$

If the initial location is not specified, the quantity $(1 /|\Omega|) \int_{\Omega} \mathbb{E}_{k, \boldsymbol{x}}[\mathcal{T}] d \boldsymbol{x}$ is regarded as the MET.

Let us introduce the pair of functions $\left(u_{1}(\boldsymbol{x}), u_{2}(\boldsymbol{x})\right)$ which is the solution of the coupled system

$$
-\left(\begin{array}{l}
1 \\
1
\end{array}\right)=a\left(\begin{array}{cc}
-1 & 1 \\
1 & -1
\end{array}\right)\left(\begin{array}{l}
u_{1}(\boldsymbol{x}) \\
u_{2}(\boldsymbol{x})
\end{array}\right)+\Delta\left(\begin{array}{l}
u_{1}(\boldsymbol{x}) \\
u_{2}(\boldsymbol{x})
\end{array}\right)
$$

with the boundary conditions

$$
\begin{aligned}
& \left.\partial_{\nu} u_{j}\right|_{\partial \Omega_{c}}=0, \quad j=1,2, \\
& \left.u_{1}\right|_{\partial \Omega_{1}}=0,\left.\quad u_{2}\right|_{\partial \Omega_{2}}=0, \\
& \left.\partial_{\nu} u_{1}\right|_{\partial \Omega_{2}}=0,\left.\quad \partial_{\nu} u_{2}\right|_{\partial \Omega_{1}}=0 .
\end{aligned}
$$

Here and throughout this paper $\partial_{\nu}$ stands for the normal derivative.

We have the following proposition for the MET:

Proposition 2.1. For $\boldsymbol{x} \in \Omega$ and $k=1,2$,

$$
\mathbb{E}_{k, \boldsymbol{x}}[\mathcal{T}]=u_{k}(\boldsymbol{x})
$$

Proof. Note that the right-hand side of the system (2.3) is the infinitesimal generator $\mathcal{A}$ of the process $\left(\sqrt{2} \boldsymbol{B}_{t}, N_{t}\right)_{t \geq 0}$. Denoting $u(\boldsymbol{x}, j)=u_{j}(\boldsymbol{x})$, we can check that, for any $s<t$ :

$$
\begin{aligned}
& \mathbb{E}\left[u\left(\boldsymbol{X}_{t \wedge \mathcal{T}}, N_{t \wedge \mathcal{T}}\right)-u\left(\boldsymbol{X}_{s \wedge \mathcal{T}}, N_{s \wedge \mathcal{T}}\right)-\int_{s \wedge \mathcal{T}}^{t \wedge \mathcal{T}} \mathcal{A} u\left(\boldsymbol{X}_{r}, N_{r}\right) d r \mid \mathcal{F}_{s \wedge \mathcal{T}}\right] \\
& =-\mathbb{E}\left[\int_{s \wedge \mathcal{T}}^{t \wedge \mathcal{T}} \partial_{\nu} u\left(\boldsymbol{X}_{r}, N_{r}\right) d L_{r} \mid \mathcal{F}_{s \wedge \mathcal{T}}\right],
\end{aligned}
$$


where $\left(\mathcal{F}_{t}\right)_{t \geq 0}$ is the natural filtration of $\left(\sqrt{2} \boldsymbol{B}_{t}, N_{t}\right)_{t \geq 0}$ and $L_{t}$ is defined as above. The Neumann boundary conditions (2.4) and (2.6) imposed on $u$ show that when the particle reaches the boundary $\partial_{\nu} u\left(\boldsymbol{X}_{r}, N_{r}\right)=0$ if $r<\mathcal{T}$. We also have $\mathcal{A} u\left(\boldsymbol{X}_{r}, N_{r}\right)=$ -1 by the partial differential equation satisfied by $u$. This shows that

$$
\mathbb{E}\left[u\left(\boldsymbol{X}_{t \wedge \mathcal{T}}, N_{t \wedge \mathcal{T}}\right)-u\left(\boldsymbol{X}_{s \wedge \mathcal{T}}, N_{s \wedge \mathcal{T}}\right)+(t \wedge \mathcal{T}-s \wedge \mathcal{T}) \mid \mathcal{F}_{s \wedge \mathcal{T}}\right]=0,
$$

i.e., the process $\left(u\left(\boldsymbol{X}_{t \wedge \mathcal{T}}, N_{t \wedge \mathcal{T}}\right)+t \wedge \mathcal{T}\right)_{t \geq 0}$ is a martingale. Applying this equality with $s=0$ and letting $t \rightarrow \infty$, we obtain

$$
\mathbb{E}\left[u\left(\boldsymbol{X}_{\mathcal{T}}, N_{\mathcal{T}}\right)-u\left(\boldsymbol{X}_{0}, N_{0}\right)+\mathcal{T} \mid \mathcal{F}_{0}\right]=0 .
$$

The Dirichlet boundary conditions (2.5) imposed on $u$ show that $u\left(\boldsymbol{X}_{\mathcal{T}}, N_{\mathcal{T}}\right)=0$. Therefore, we have

$$
\mathbb{E}\left[\mathcal{T} \mid \mathcal{F}_{0}\right]=\mathbb{E}\left[u\left(\boldsymbol{X}_{0}, N_{0}\right) \mid \mathcal{F}_{0}\right] .
$$

This gives the desired result when the initial distribution is such that $N_{0}=k$ and $\boldsymbol{X}_{0}=\boldsymbol{x}$.

3. The Neumann functions and integral equations. Let $N(\boldsymbol{x}, \boldsymbol{z})$ be the Neumann function for $-\Delta$ in $\Omega$ with a Dirac mass at $z \in \Omega$ :

$$
\left\{\begin{array}{l}
\Delta_{\boldsymbol{x}} N=-\delta_{\boldsymbol{z}}, \quad \boldsymbol{x} \in \Omega, \\
\left.\partial_{\nu} N\right|_{\partial \Omega}=-\frac{1}{|\partial \Omega|}, \quad \int_{\partial \Omega} N(\boldsymbol{x}, \boldsymbol{z}) d \sigma(\boldsymbol{x})=0
\end{array}\right.
$$

If $\boldsymbol{z} \in \partial \Omega$, then the Neumann function, which we denote by $N_{\partial \Omega}(\boldsymbol{x}, \boldsymbol{z})$, can be expanded as

$$
N_{\partial \Omega}(\boldsymbol{x}, \boldsymbol{z})=-\frac{1}{\pi} \ln |\boldsymbol{x}-\boldsymbol{z}|+R(\boldsymbol{x}, \boldsymbol{z}),
$$

where $R(\cdot, \boldsymbol{z})$ belongs to $H^{3 / 2}(\Omega)$, the standard Sobolev space of order $3 / 2$, uniformly in $\boldsymbol{z} \in \partial \Omega$, see [1].

For a positive constant $a$, let $M^{a}(\boldsymbol{x}, \boldsymbol{z})$ be the Neumann function for $-\Delta+2 a$ in $\Omega$ with a Dirac mass at $z \in \Omega$ :

$$
\left\{\begin{array}{l}
\Delta_{\boldsymbol{x}} M^{a}-2 a M^{a}=-\delta_{\boldsymbol{z}}, \quad \boldsymbol{x} \in \Omega \\
\left.\partial_{\nu} M^{a}\right|_{\partial \Omega}=0
\end{array}\right.
$$

Note that we can write $M^{a}(\boldsymbol{x}, \boldsymbol{z})$ as

$$
M^{a}(\boldsymbol{x}, \boldsymbol{z})=\sum_{j=0}^{\infty} \frac{\phi_{j}(\boldsymbol{x}) \phi_{j}(\boldsymbol{z})}{\lambda_{j}+2 a},
$$

where $\left(\lambda_{j}, \phi_{j}\right)_{j \geq 0}$ are pairs of eigenvalues and corresponding (normalized) eigenvectors of $-\Delta$ over $\Omega$ with Neumann boundary conditions on $\partial \Omega$. In particular, $\lambda_{0}=0$ and $\phi_{0}=1 / \sqrt{|\Omega|}$. It then follows that $[2]$

$$
\int_{\Omega} M^{a}(\boldsymbol{x}, \boldsymbol{z}) d \boldsymbol{x}=\frac{1}{2 a} .
$$


If $\boldsymbol{z} \in \partial \Omega$, then $M_{\partial \Omega}^{a}(\boldsymbol{x}, \boldsymbol{z})$ can be expanded as

$$
M_{\partial \Omega}^{a}(\boldsymbol{x}, \boldsymbol{z})=-\frac{1}{\pi} \ln |\boldsymbol{x}-\boldsymbol{z}|+R_{a}(\boldsymbol{x}, \boldsymbol{z}),
$$

where $R_{a}(\cdot, \boldsymbol{z})$ also belongs to $H^{3 / 2}(\Omega)$ uniformly in $z \in \partial \Omega$. We emphasize that $R$ and $R_{a}$ are symmetric in their arguments.

Let us introduce

$$
g(\boldsymbol{z})=\int_{\Omega} N(\boldsymbol{x}, \boldsymbol{z}) d \boldsymbol{x}, \quad \boldsymbol{z} \in \Omega .
$$

Let $\left(u_{1}(\boldsymbol{x}), u_{2}(\boldsymbol{x})\right)$ be the solution to (2.3). One can use Green's formula, (2.4), and (2.6) to show that the following representations hold for all $\boldsymbol{z} \in \Omega$ :

$$
\begin{aligned}
u_{1}(\boldsymbol{z})=g(\boldsymbol{z}) & +\frac{C}{2}+\int_{\partial \Omega_{1}} \partial_{\nu} u_{1}(\boldsymbol{x}) \frac{N_{\partial \Omega}+M_{\partial \Omega}}{2}(\boldsymbol{z}, \boldsymbol{x}) d \sigma(\boldsymbol{x}) \\
& +\int_{\partial \Omega_{2}} \partial_{\nu} u_{2}(\boldsymbol{x}) \frac{N_{\partial \Omega}-M_{\partial \Omega}}{2}(\boldsymbol{z}, \boldsymbol{x}) d \sigma(\boldsymbol{x}),
\end{aligned}
$$

and

$$
\begin{aligned}
u_{2}(\boldsymbol{z})=g & (\boldsymbol{z})+\frac{C}{2}+\int_{\partial \Omega_{2}} \partial_{\nu} u_{2}(\boldsymbol{x}) \frac{N_{\partial \Omega}+M_{\partial \Omega}}{2}(\boldsymbol{z}, \boldsymbol{x}) d \sigma(\boldsymbol{x}) \\
& +\int_{\partial \Omega_{1}} \partial_{\nu} u_{1}(\boldsymbol{x}) \frac{N_{\partial \Omega}-M_{\partial \Omega}}{2}(\boldsymbol{z}, \boldsymbol{x}) d \sigma(\boldsymbol{x}),
\end{aligned}
$$

where $C$ is the constant defined by

$$
C=\frac{1}{|\partial \Omega|} \int_{\partial \Omega}\left(u_{1}(\boldsymbol{x})+u_{2}(\boldsymbol{x})\right) d \sigma(\boldsymbol{x}) .
$$

In view of (2.5), we have for all $\boldsymbol{z} \in \partial \Omega_{1}$

$$
\begin{aligned}
& g(\boldsymbol{z})+\frac{C}{2}-\frac{1}{\pi} \int_{\partial \Omega_{1}} \partial_{\nu} u_{1}(\boldsymbol{x}) \ln |\boldsymbol{x}-\boldsymbol{z}| d \sigma(\boldsymbol{x}) \\
& +\int_{\partial \Omega_{1}} \partial_{\nu} u_{1}(\boldsymbol{x}) \frac{R+R_{a}}{2}(\boldsymbol{z}, \boldsymbol{x}) d \sigma(\boldsymbol{x})+\int_{\partial \Omega_{2}} \partial_{\nu} u_{2}(\boldsymbol{x}) \frac{R-R_{a}}{2}(\boldsymbol{z}, \boldsymbol{x}) d \sigma(\boldsymbol{x})=0,
\end{aligned}
$$

and for all $\boldsymbol{z} \in \partial \Omega_{2}$

$$
\begin{aligned}
& g(\boldsymbol{z})+\frac{C}{2}-\frac{1}{\pi} \int_{\partial \Omega_{2}} \partial_{\nu} u_{2}(\boldsymbol{x}) \ln |\boldsymbol{x}-\boldsymbol{z}| d \sigma(\boldsymbol{x}) \\
& +\int_{\partial \Omega_{2}} \partial_{\nu} u_{2}(\boldsymbol{x}) \frac{R+R_{a}}{2}(\boldsymbol{z}, \boldsymbol{x}) d \sigma(\boldsymbol{x})+\int_{\partial \Omega_{1}} \partial_{\nu} u_{1}(\boldsymbol{x}) \frac{R-R_{a}}{2}(\boldsymbol{z}, \boldsymbol{x}) d \sigma(\boldsymbol{x})=0 .
\end{aligned}
$$

We solve the integral equations (3.10) and (3.11) asymptotically for $\partial_{\nu} u_{1}$ and $\partial_{\nu} u_{1}$ in the next section.

4. Asymptotic expansions. We parameterize $\partial \Omega_{j}$ by arclength:

$$
\partial \Omega_{j}=\left\{\boldsymbol{x}_{j}(t), t \in\left[-\varepsilon_{j}, \varepsilon_{j}\right]\right\},
$$

with $\varepsilon_{j} \ll 1, j=1,2$. Let $\boldsymbol{x}_{j}^{*}=\boldsymbol{x}_{j}(0)$ be the centers of the arcs. 
Define for $j=1,2$

$$
\phi_{j}(t)=\varepsilon_{j} \partial_{\nu} u_{j}\left(\boldsymbol{x}_{j}\left(\varepsilon_{j} t\right)\right), \quad t \in[-1,1] .
$$

We then define the operator $\mathcal{L}$ from $\mathbf{X}=\left\{\phi, \int_{-1}^{1} \sqrt{1-s^{2}} \phi(s)^{2} d s<\infty\right\}$ to $\mathbf{Y}=\{\phi \in$ $\left.\mathcal{C}(-1,1), \phi^{\prime} \in \mathbf{X}\right\}$ by

$$
\mathcal{L}[\phi](t)=\int_{-1}^{1} \ln |t-s| \phi(s) d s .
$$

It can be shown, see for instance [2], that $\mathcal{L}: \mathbf{X} \rightarrow \mathbf{Y}$ is invertible and

$$
\mathcal{L}^{-1}[1](t)=-\frac{1}{\pi \ln 2} \frac{1}{\sqrt{1-t^{2}}} .
$$

After scaling, (3.10) and (3.11) take the forms

$$
\begin{aligned}
\frac{1}{\pi} \mathcal{L}\left[\phi_{1}\right](t)+\varepsilon \mathcal{K}_{11}\left[\phi_{1}\right](t)+\varepsilon \mathcal{K}_{12}\left[\phi_{2}\right](t)= & \left(-\frac{\ln \varepsilon_{1}}{\pi}+\frac{R+R_{a}}{2}\left(\boldsymbol{x}_{1}^{*}, \boldsymbol{x}_{1}^{*}\right)\right) \int_{-1}^{1} \phi_{1}(s) d s \\
& +\frac{R-R_{a}}{2}\left(\boldsymbol{x}_{2}^{*}, \boldsymbol{x}_{1}^{*}\right) \int_{-1}^{1} \phi_{2}(s) d s+g\left(\boldsymbol{x}_{1}(\varepsilon t)\right)+\frac{C}{2}, \\
\frac{1}{\pi} \mathcal{L}\left[\phi_{2}\right](t)+\varepsilon \mathcal{K}_{21}\left[\phi_{1}\right](t)+\varepsilon \mathcal{K}_{22}\left[\phi_{2}\right](t)= & \left(-\frac{\ln \varepsilon_{2}}{\pi}+\frac{R+R_{a}}{2}\left(\boldsymbol{x}_{2}^{*}, \boldsymbol{x}_{2}^{*}\right)\right) \int_{-1}^{1} \phi_{2}(s) d s \\
& +\frac{R-R_{a}}{2}\left(\boldsymbol{x}_{1}^{*}, \boldsymbol{x}_{2}^{*}\right) \int_{-1}^{1} \phi_{1}(s) d s+g\left(\boldsymbol{x}_{2}(\varepsilon t)\right)+\frac{C}{2},
\end{aligned}
$$

where the operators $\mathcal{K}_{j k}$ are defined by

$$
\begin{aligned}
\mathcal{K}_{j j}[\phi](t)= & \frac{1}{\pi \varepsilon} \int_{-1}^{1} \ln \left(\frac{\left|\boldsymbol{x}_{j}(\varepsilon t)-\boldsymbol{x}_{j}(\varepsilon s)\right|}{\varepsilon|t-s|}\right) \phi(s) d s \\
& -\frac{1}{\varepsilon} \int_{-1}^{1}\left(\frac{R+R_{a}}{2}\left(\boldsymbol{x}_{j}(\varepsilon t), \boldsymbol{x}_{j}(\varepsilon s)\right)-\frac{R+R_{a}}{2}\left(\boldsymbol{x}_{j}^{*}, \boldsymbol{x}_{j}^{*}\right)\right) \phi(s) d s, \\
\mathcal{K}_{12}[\phi](t)= & -\frac{1}{\varepsilon} \int_{-1}^{1}\left(\frac{R-R_{a}}{2}\left(\boldsymbol{x}_{1}(\varepsilon t), \boldsymbol{x}_{2}(\varepsilon s)\right)-\frac{R-R_{a}}{2}\left(\boldsymbol{x}_{1}^{*}, \boldsymbol{x}_{2}^{*}\right)\right) \phi(s) d s, \\
\mathcal{K}_{21}[\phi](t)= & -\frac{1}{\varepsilon} \int_{-1}^{1}\left(\frac{R-R_{a}}{2}\left(\boldsymbol{x}_{2}(\varepsilon t), \boldsymbol{x}_{1}(\varepsilon s)\right)-\frac{R-R_{a}}{2}\left(\boldsymbol{x}_{2}^{*}, \boldsymbol{x}_{1}^{*}\right)\right) \phi(s) d s .
\end{aligned}
$$

We emphasize that $\mathcal{K}_{j k}$ is a bounded operator from $\mathbf{X}$ into $\mathbf{Y}$ independently of $\varepsilon$ since $\boldsymbol{x}_{j}$ is $\mathcal{C}^{2}$ and $R, R_{a}$ are in $H^{3 / 2}$. Denoting

$$
A_{j}=\int_{-1}^{1} \phi_{j}(s) d s=\int_{\partial \Omega_{j}} \partial_{\nu} u_{j}(\boldsymbol{x}) d \sigma(\boldsymbol{x}), \quad j=1,2,
$$

we can rewrite the equations in the form

$$
\frac{1}{\pi}\left(\begin{array}{cc}
\mathcal{L} & 0 \\
0 & \mathcal{L}
\end{array}\right)\left(\begin{array}{l}
\phi_{1} \\
\phi_{2}
\end{array}\right)(t)+\varepsilon\left(\begin{array}{ll}
\mathcal{K}_{11} & \mathcal{K}_{12} \\
\mathcal{K}_{21} & \mathcal{K}_{22}
\end{array}\right)\left(\begin{array}{l}
\phi_{1} \\
\phi_{2}
\end{array}\right)(t)=\left(\begin{array}{l}
q_{1} \\
q_{2}
\end{array}\right)+O(\varepsilon), \quad t \in[-1,1],
$$

where

$$
\begin{aligned}
& q_{1}=g\left(\boldsymbol{x}_{1}^{*}\right)+\frac{C}{2}+\left(-\frac{\ln \varepsilon_{1}}{\pi}+\frac{R+R_{a}}{2}\left(\boldsymbol{x}_{1}^{*}, \boldsymbol{x}_{1}^{*}\right)\right) A_{1}+\frac{R-R_{a}}{2}\left(\boldsymbol{x}_{2}^{*}, \boldsymbol{x}_{1}^{*}\right) A_{2}, \\
& q_{2}=g\left(\boldsymbol{x}_{2}^{*}\right)+\frac{C}{2}+\left(-\frac{\ln \varepsilon_{2}}{\pi}+\frac{R+R_{a}}{2}\left(\boldsymbol{x}_{2}^{*}, \boldsymbol{x}_{2}^{*}\right)\right) A_{2}+\frac{R-R_{a}}{2}\left(\boldsymbol{x}_{1}^{*}, \boldsymbol{x}_{2}^{*}\right) A_{1} .
\end{aligned}
$$


It then follows from (4.3) that, for $t \in[-1,1]$,

$$
\phi_{j}(t)=\pi q_{j} \mathcal{L}^{-1}[1](t)=-\frac{q_{j}}{\ln 2} \frac{1}{\sqrt{1-t^{2}}}+O(\varepsilon), \quad j=1,2 .
$$

Integrating this identity in $t$ we find

$$
\begin{aligned}
& g\left(\boldsymbol{x}_{1}^{*}\right)+\frac{C}{2}+\left(-\frac{\ln \varepsilon_{1} / 2}{\pi}+\frac{R+R_{a}}{2}\left(\boldsymbol{x}_{1}^{*}, \boldsymbol{x}_{1}^{*}\right)\right) A_{1}+\frac{R-R_{a}}{2}\left(\boldsymbol{x}_{2}^{*}, \boldsymbol{x}_{1}^{*}\right) A_{2}=O(\varepsilon), \\
& g\left(\boldsymbol{x}_{2}^{*}\right)+\frac{C}{2}+\left(-\frac{\ln \varepsilon_{2} / 2}{\pi}+\frac{R+R_{a}}{2}\left(\boldsymbol{x}_{2}^{*}, \boldsymbol{x}_{2}^{*}\right)\right) A_{2}+\frac{R-R_{a}}{2}\left(\boldsymbol{x}_{1}^{*}, \boldsymbol{x}_{2}^{*}\right) A_{1}=O(\varepsilon) .
\end{aligned}
$$

Moreover, we have the compatibility condition

$$
A_{1}+A_{2}=-2|\Omega| .
$$

which can be obtained by integrating $\Delta\left(u_{1}+u_{2}\right)$ over $\Omega$.

The equations (4.4-4.6) determine the value of $C$ up to terms of order $\varepsilon$ :

$$
C=\frac{C_{n}}{C_{d}}
$$

where

$$
\begin{aligned}
C_{n}= & \frac{2|\Omega|}{\pi^{2}} \ln \frac{2}{\varepsilon_{1}} \ln \frac{2}{\varepsilon_{2}} \\
& +\frac{1}{\pi} \ln \frac{2}{\varepsilon_{1}}\left(|\Omega|\left(R+R_{a}\right)\left(\boldsymbol{x}_{2}^{*}, \boldsymbol{x}_{2}^{*}\right)-g\left(\boldsymbol{x}_{2}^{*}\right)\right) \\
& +\frac{1}{\pi} \ln \frac{2}{\varepsilon_{2}}\left(|\Omega|\left(R+R_{a}\right)\left(\boldsymbol{x}_{1}^{*}, \boldsymbol{x}_{1}^{*}\right)-g\left(\boldsymbol{x}_{1}^{*}\right)\right) \\
& -g\left(\boldsymbol{x}_{1}^{*}\right)\left(\frac{R+R_{a}}{2}\left(\boldsymbol{x}_{2}^{*}, \boldsymbol{x}_{2}^{*}\right)-\frac{R-R_{a}}{2}\left(\boldsymbol{x}_{1}^{*}, \boldsymbol{x}_{2}^{*}\right)\right) \\
& -g\left(\boldsymbol{x}_{2}^{*}\right)\left(\frac{R+R_{a}}{2}\left(\boldsymbol{x}_{1}^{*}, \boldsymbol{x}_{1}^{*}\right)-\frac{R-R_{a}}{2}\left(\boldsymbol{x}_{1}^{*}, \boldsymbol{x}_{2}^{*}\right)\right) \\
& +\frac{R+R_{a}}{2}\left(\boldsymbol{x}_{2}^{*}, \boldsymbol{x}_{2}^{*}\right) \frac{R+R_{a}}{2}\left(\boldsymbol{x}_{1}^{*}, \boldsymbol{x}_{1}^{*}\right)-\frac{R-R_{a}}{2}\left(\boldsymbol{x}_{1}^{*}, \boldsymbol{x}_{2}^{*}\right)^{2}, \\
C_{d}= & \frac{1}{2 \pi}\left(\ln \frac{2}{\varepsilon_{1}}+\ln \frac{2}{\varepsilon_{2}}\right) \\
& +\frac{R+R_{a}}{4}\left(\boldsymbol{x}_{1}^{*}, \boldsymbol{x}_{1}^{*}\right)+\frac{R+R_{a}}{4}\left(\boldsymbol{x}_{2}^{*}, \boldsymbol{x}_{2}^{*}\right)-\frac{R-R_{a}}{2}\left(\boldsymbol{x}_{1}^{*}, \boldsymbol{x}_{2}^{*}\right) .
\end{aligned}
$$

They also determine the value of $A_{j}$ up to terms of order $\varepsilon$ :

$$
A_{j}=-\frac{g\left(\boldsymbol{x}_{j}^{*}\right)+\frac{C}{2}-|\Omega|\left(R-R_{a}\right)\left(\boldsymbol{x}_{1}^{*}, \boldsymbol{x}_{2}^{*}\right)}{\frac{1}{\pi} \ln \frac{2}{\varepsilon_{j}}+\frac{R+R_{a}}{2}\left(\boldsymbol{x}_{j}^{*}, \boldsymbol{x}_{j}^{*}\right)-\frac{R-R_{a}}{2}\left(\boldsymbol{x}_{1}^{*}, \boldsymbol{x}_{2}^{*}\right)}, \quad j=1,2 .
$$

In view of (3.7) and (3.8), we have

$$
\begin{aligned}
& \mathbb{E}_{1, \boldsymbol{x}}[\mathcal{T}]=g(\boldsymbol{x})+\frac{C}{2}+\frac{A_{1}}{2}\left(N_{\partial \Omega}+M_{\partial \Omega}\right)\left(\boldsymbol{x}, \boldsymbol{x}_{1}^{*}\right)+\frac{A_{2}}{2}\left(N_{\partial \Omega}-M_{\partial \Omega}\right)\left(\boldsymbol{x}, \boldsymbol{x}_{2}^{*}\right), \\
& \mathbb{E}_{2, \boldsymbol{x}}[\mathcal{T}]=g(\boldsymbol{x})+\frac{C}{2}+\frac{A_{2}}{2}\left(N_{\partial \Omega}+M_{\partial \Omega}\right)\left(\boldsymbol{x}, \boldsymbol{x}_{2}^{*}\right)+\frac{A_{1}}{2}\left(N_{\partial \Omega}-M_{\partial \Omega}\right)\left(\boldsymbol{x}, \boldsymbol{x}_{1}^{*}\right),
\end{aligned}
$$


up to terms of order $\varepsilon$ as long as $\boldsymbol{x}$ is away from $\partial \Omega_{1}$ and $\partial \Omega_{2}$. If the initial distribution of $N_{0}$ is uniform over $\{1,2\}$, then we have

$$
\mathbb{E}_{u, \boldsymbol{x}}[\mathcal{T}]=g(\boldsymbol{x})+\frac{C}{2}+\frac{A_{1}}{2} N_{\partial \Omega}\left(\boldsymbol{x}, \boldsymbol{x}_{1}^{*}\right)+\frac{A_{2}}{2} N_{\partial \Omega}\left(\boldsymbol{x}, \boldsymbol{x}_{2}^{*}\right)
$$

Asymptotic expansions of the MET can be derived using (4.7-4.10), which we do in the next section.

5. Role of the switching rate. In this section we study the expressions (4.114.13 ) and we show that the leading order term (of order $\ln \left(2 / \varepsilon_{j}\right)$ ) of the MET is independent of the switching rate $a$, while the first-order correction term (of order one) depends on $a$ and is a decaying function of $a$.

5.1. Leading-order term. We find from (4.7-4.9) that the leading-order term of $C$ is independent of $a$ and $\boldsymbol{x}$ :

$$
C=\frac{4|\Omega|}{\pi} \frac{\ln \frac{2}{\varepsilon_{1}} \ln \frac{2}{\varepsilon_{2}}}{\ln \frac{2}{\varepsilon_{1}}+\ln \frac{2}{\varepsilon_{2}}}+O(1) .
$$

Since

$$
A_{j}=-\frac{\pi C}{2 \ln \frac{2}{\varepsilon_{j}}}+O(1)
$$

which can be seen from (4.10), one can see that the leading-order term of the MET is also independent of $a$ :

$$
\mathbb{E}_{k, \boldsymbol{x}}[\mathcal{T}]=\frac{2|\Omega|}{\pi} \frac{\ln \frac{2}{\varepsilon_{1}} \ln \frac{2}{\varepsilon_{2}}}{\ln \frac{2}{\varepsilon_{1}}+\ln \frac{2}{\varepsilon_{2}}}+O(1) .
$$

It is worth emphasizing that it is also independent of the initial state $N_{0}$ and position $\boldsymbol{x}$.

It is quite interesting to notice that the number

$$
\frac{|\Omega|}{\pi} \frac{\ln \frac{2}{\varepsilon_{1}} \ln \frac{2}{\varepsilon_{2}}}{\ln \frac{2}{\varepsilon_{1}}+\ln \frac{2}{\varepsilon_{2}}}
$$

is the leading order term of the MET when there are two (well separated) gates of length $\varepsilon_{1}$ and $\varepsilon_{2}$ which opens all time (see [3, Theorem 3.3] for a proof when the domain is a circle). Therefore (5.2) shows that the leading order term in the MET with two switching gates is exactly twice the one with two time-independent gates. In this scaling regime with small gates and when the gates are open only half the time, the time to find a gate doubles to leading order.

5.2. First-order correction. We can expand the expressions for $C, A_{1}$, and $A_{2}$ in powers of $\ln \left(2 / \varepsilon_{j}\right)$ and taking into account the terms of order $\ln \left(2 / \varepsilon_{j}\right)$ and of 
order one. We find that

$$
\begin{aligned}
C= & \frac{4|\Omega|}{\pi} \frac{\ln \frac{2}{\varepsilon_{1}} \ln \frac{2}{\varepsilon_{2}}}{\ln \frac{2}{\varepsilon_{1}}+\ln \frac{2}{\varepsilon_{2}}} \\
& +2|\Omega| \frac{\left(\ln \frac{2}{\varepsilon_{2}}\right)^{2}}{\left(\ln \frac{2}{\varepsilon_{1}}+\ln \frac{2}{\varepsilon_{2}}\right)^{2}}\left(R+R_{a}\right)\left(\boldsymbol{x}_{1}^{*}, \boldsymbol{x}_{1}^{*}\right)+2|\Omega| \frac{\left(\ln \frac{2}{\varepsilon_{1}}\right)^{2}}{\left(\ln \frac{2}{\varepsilon_{1}}+\ln \frac{2}{\varepsilon_{2}}\right)^{2}}\left(R+R_{a}\right)\left(\boldsymbol{x}_{2}^{*}, \boldsymbol{x}_{2}^{*}\right) \\
& +4|\Omega| \frac{\left(\ln \frac{2}{\varepsilon_{1}}\right)\left(\ln \frac{2}{\varepsilon_{2}}\right)}{\left(\ln \frac{2}{\varepsilon_{1}}+\ln \frac{2}{\varepsilon_{2}}\right)^{2}}\left(R-R_{a}\right)\left(\boldsymbol{x}_{1}^{*}, \boldsymbol{x}_{2}^{*}\right) \\
& +2 \frac{\ln \frac{2}{\varepsilon_{2}}}{\ln \frac{2}{\varepsilon_{1}}+\ln \frac{2}{\varepsilon_{2}}} g\left(\boldsymbol{x}_{1}^{*}\right)+2 \frac{\ln \frac{2}{\varepsilon_{1}}}{\ln \frac{2}{\varepsilon_{1}}+\ln \frac{2}{\varepsilon_{2}}} g\left(\boldsymbol{x}_{2}^{*}\right)+O\left(\frac{1}{\ln \frac{2}{\varepsilon_{1}}+\ln \frac{2}{\varepsilon_{2}}}\right)
\end{aligned}
$$

and

$$
A_{1}=-2|\Omega| \frac{\ln \frac{2}{\varepsilon_{2}}}{\ln \frac{2}{\varepsilon_{1}}+\ln \frac{2}{\varepsilon_{2}}}+O\left(\frac{1}{\ln \frac{2}{\varepsilon_{1}}}\right), \quad A_{2}=-2|\Omega| \frac{\ln \frac{2}{\varepsilon_{1}}}{\ln \frac{2}{\varepsilon_{1}}+\ln \frac{2}{\varepsilon_{2}}}+O\left(\frac{1}{\ln \frac{2}{\varepsilon_{2}}}\right) .
$$

This gives for the MET

$$
\begin{aligned}
\mathbb{E}_{1, \boldsymbol{x}}[\mathcal{T}]= & g(\boldsymbol{x})+\frac{C}{2}-|\Omega| \frac{\ln \frac{2}{\varepsilon_{2}}}{\ln \frac{2}{\varepsilon_{1}}+\ln \frac{2}{\varepsilon_{2}}}\left(N_{\partial \Omega}+M_{\partial \Omega}\right)\left(\boldsymbol{x}, \boldsymbol{x}_{1}^{*}\right) \\
& -|\Omega| \frac{\ln \frac{2}{\varepsilon_{1}}}{\ln \frac{2}{\varepsilon_{1}}+\ln \frac{2}{\varepsilon_{2}}}\left(N_{\partial \Omega}-M_{\partial \Omega}\right)\left(\boldsymbol{x}, \boldsymbol{x}_{2}^{*}\right)+O\left(\frac{1}{\ln \frac{2}{\varepsilon_{1}}+\ln \frac{2}{\varepsilon_{2}}}\right), \\
\mathbb{E}_{2, \boldsymbol{x}}[\mathcal{T}]= & g(\boldsymbol{x})+\frac{C}{2}-|\Omega| \frac{\ln \frac{2}{\varepsilon_{1}}}{\ln \frac{2}{\varepsilon_{1}}+\ln \frac{2}{\varepsilon_{2}}}\left(N_{\partial \Omega}+M_{\partial \Omega}\right)\left(\boldsymbol{x}, \boldsymbol{x}_{2}^{*}\right) \\
& -|\Omega| \frac{\ln \frac{2}{\varepsilon_{2}}}{\ln \frac{2}{\varepsilon_{1}}+\ln \frac{2}{\varepsilon_{2}}}\left(N_{\partial \Omega}-M_{\partial \Omega}\right)\left(\boldsymbol{x}, \boldsymbol{x}_{1}^{*}\right)+O\left(\frac{1}{\ln \frac{2}{\varepsilon_{1}}+\ln \frac{2}{\varepsilon_{2}}}\right),
\end{aligned}
$$

and finally

$$
\begin{aligned}
\mathbb{E}_{u, \boldsymbol{x}}[\mathcal{T}]= & g(\boldsymbol{x})+\frac{C}{2}-|\Omega| \frac{\ln \frac{2}{\varepsilon_{1}}}{\ln \frac{2}{\varepsilon_{1}}+\ln \frac{2}{\varepsilon_{2}}} N_{\partial \Omega}\left(\boldsymbol{x}, \boldsymbol{x}_{2}^{*}\right) \\
& -|\Omega| \frac{\ln \frac{2}{\varepsilon_{2}}}{\ln \frac{2}{\varepsilon_{1}}+\ln \frac{2}{\varepsilon_{2}}} N_{\partial \Omega}\left(\boldsymbol{x}, \boldsymbol{x}_{1}^{*}\right)+O\left(\frac{1}{\ln \frac{2}{\varepsilon_{1}}+\ln \frac{2}{\varepsilon_{2}}}\right) .
\end{aligned}
$$

The terms of order one depend on $a$ through the terms $C$ and $M_{\partial \Omega}$. We have, up to terms of order $O\left(\left(\ln \frac{2}{\varepsilon_{1}}+\ln \frac{2}{\varepsilon_{2}}\right)^{-1}\right)$ :

$$
\begin{aligned}
\frac{\partial C}{\partial a}= & 2|\Omega| \frac{\left(\ln \frac{2}{\varepsilon_{2}}\right)^{2}}{\left(\ln \frac{2}{\varepsilon_{1}}+\ln \frac{2}{\varepsilon_{2}}\right)^{2}} \frac{\partial R_{a}\left(\boldsymbol{x}_{1}^{*}, \boldsymbol{x}_{1}^{*}\right)}{\partial a}+2|\Omega| \frac{\left(\ln \frac{2}{\varepsilon_{1}}\right)^{2}}{\left(\ln \frac{2}{\varepsilon_{1}}+\ln \frac{2}{\varepsilon_{2}}\right)^{2}} \frac{\partial R_{a}\left(\boldsymbol{x}_{2}^{*}, \boldsymbol{x}_{2}^{*}\right)}{\partial a} \\
& -4|\Omega| \frac{\left(\ln \frac{2}{\varepsilon_{1}}\right)\left(\ln \frac{2}{\varepsilon_{2}}\right)}{\left(\ln \frac{2}{\varepsilon_{1}}+\ln \frac{2}{\varepsilon_{2}}\right)^{2}} \frac{\partial R_{a}\left(\boldsymbol{x}_{1}^{*}, \boldsymbol{x}_{2}^{*}\right)}{\partial a} .
\end{aligned}
$$

The following lemma applied with $p=\ln \left(2 / \varepsilon_{2}\right) /\left[\ln \left(2 / \varepsilon_{2}\right)+\ln \left(2 / \varepsilon_{1}\right)\right]$ shows that

$$
\frac{\partial C}{\partial a}<0
$$


provided that $\varepsilon_{j}$ is small enough.

LEMma 5.1. We have the equality

$$
\frac{\partial R_{a}\left(\boldsymbol{x}_{j}^{*}, \boldsymbol{x}_{k}^{*}\right)}{\partial a}=-2 \int_{\Omega} M_{\partial \Omega}\left(\boldsymbol{x}_{j}^{*}, \boldsymbol{x}\right) M_{\partial \Omega}\left(\boldsymbol{x}_{k}^{*}, \boldsymbol{x}\right) d \boldsymbol{x}, \quad j, k=1,2 .
$$

Moreover, for any $p \in(0,1)$ we have the inequality

$$
\frac{p^{2}}{2} \frac{\partial R_{a}\left(\boldsymbol{x}_{1}^{*}, \boldsymbol{x}_{1}^{*}\right)}{\partial a}+\frac{(1-p)^{2}}{2} \frac{\partial R_{a}\left(\boldsymbol{x}_{2}^{*}, \boldsymbol{x}_{2}^{*}\right)}{\partial a}-p(1-p) \frac{\partial R_{a}\left(\boldsymbol{x}_{1}^{*}, \boldsymbol{x}_{2}^{*}\right)}{\partial a}<0 .
$$

Proof. We denote $M^{\prime}(\boldsymbol{x}, \boldsymbol{z}):=\partial_{a} M(\boldsymbol{x}, \boldsymbol{z})$ for $\boldsymbol{z} \in \Omega$. It is the solution to the problem

$$
\Delta_{\boldsymbol{x}} M^{\prime}(\boldsymbol{x}, \boldsymbol{z})-2 a M^{\prime}(\boldsymbol{x}, \boldsymbol{z})=2 M(\boldsymbol{x}, \boldsymbol{z}),\left.\quad \partial_{\nu} M^{\prime}(\cdot, \boldsymbol{z})\right|_{\partial \Omega}=0 .
$$

Thus we obtain by Green's formula

$$
M^{\prime}(\boldsymbol{x}, \boldsymbol{z})=-2 \int_{\Omega} M(\boldsymbol{x}, \boldsymbol{y}) M(\boldsymbol{y}, \boldsymbol{z}) d \boldsymbol{y} .
$$

Since $\partial_{a} R_{a}\left(\boldsymbol{x}, \boldsymbol{x}_{j}^{*}\right)=\partial_{a} M_{\partial \Omega}\left(\boldsymbol{x}, \boldsymbol{x}_{j}^{*}\right)$, we obtain (5.7).

From Cauchy-Schwarz inequality, we have

$$
\begin{aligned}
\left|p(1-p) \frac{\partial R_{a}\left(\boldsymbol{x}_{1}^{*}, \boldsymbol{x}_{2}^{*}\right)}{\partial a}\right| & =\left|2 \int_{\Omega} p M_{\partial \Omega}\left(\boldsymbol{x}_{1}^{*}, \boldsymbol{x}\right)(1-p) M_{\partial \Omega}\left(\boldsymbol{x}_{2}^{*}, \boldsymbol{x}\right) d \boldsymbol{x}\right| \\
& <\left[2 p^{2} \int_{\Omega} M_{\partial \Omega}\left(\boldsymbol{x}_{1}^{*}, \boldsymbol{x}\right)^{2} d \boldsymbol{x}\right]^{1 / 2}\left[2(1-p)^{2} \int_{\Omega} M_{\partial \Omega}\left(\boldsymbol{x}_{2}^{*}, \boldsymbol{x}\right)^{2} d \boldsymbol{x}\right]^{1 / 2} .
\end{aligned}
$$

The inequality is strict since $\boldsymbol{x}_{1}^{*} \neq \boldsymbol{x}_{2}^{*}$ and therefore we cannot have $M_{\partial \Omega}\left(\boldsymbol{x}_{2}^{*}, \boldsymbol{x}\right)=$ $M_{\partial \Omega}\left(\boldsymbol{x}_{1}^{*}, \boldsymbol{x}\right)$ for almost every $\boldsymbol{x} \in \Omega$. Using the inequality $\alpha \beta \leq(1 / 2) \alpha^{2}+(1 / 2) \beta^{2}$, we obtain (5.8).

We have, up to terms of order $O\left(\left(\ln \left(2 / \varepsilon_{1}\right)+\ln \left(2 / \varepsilon_{2}\right)\right)^{-1}\right)$

$$
\begin{aligned}
& \frac{\partial \mathbb{E}_{1, \boldsymbol{x}}[\mathcal{T}]}{\partial a}=\frac{1}{2} \frac{\partial C}{\partial a}-|\Omega| \frac{\ln \frac{2}{\varepsilon_{2}}}{\ln \frac{2}{\varepsilon_{1}}+\ln \frac{2}{\varepsilon_{2}}} \frac{\partial R_{a}\left(\boldsymbol{x}, \boldsymbol{x}_{1}^{*}\right)}{\partial a}+|\Omega| \frac{\ln \frac{2}{\varepsilon_{1}}}{\ln \frac{2}{\varepsilon_{1}}+\ln \frac{2}{\varepsilon_{2}}} \frac{\partial R_{a}\left(\boldsymbol{x}, \boldsymbol{x}_{2}^{*}\right)}{\partial a} \\
& \frac{\partial \mathbb{E}_{2, \boldsymbol{x}}[\mathcal{T}]}{\partial a}=\frac{1}{2} \frac{\partial C}{\partial a}-|\Omega| \frac{\ln \frac{2}{\varepsilon_{1}}}{\ln \frac{2}{\varepsilon_{1}}+\ln \frac{2}{\varepsilon_{2}}} \frac{\partial R_{a}\left(\boldsymbol{x}, \boldsymbol{x}_{2}^{*}\right)}{\partial a}+|\Omega| \frac{\ln \frac{2}{\varepsilon_{2}}}{\ln \frac{2}{\varepsilon_{1}}+\ln \frac{2}{\varepsilon_{2}}} \frac{\partial R_{a}\left(\boldsymbol{x}, \boldsymbol{x}_{1}^{*}\right)}{\partial a}
\end{aligned}
$$

and finally

$$
\frac{\partial \mathbb{E}_{u, \boldsymbol{x}}[\mathcal{T}]}{\partial a}=\frac{1}{2} \frac{\partial C}{\partial a} .
$$

The last result allows us to conclude that the MET decays with the switching rate whatever the initial starting point $\boldsymbol{x}$, since we have for $\varepsilon_{j}$ small enough

$$
\frac{\partial \mathbb{E}_{u, \boldsymbol{x}}[\mathcal{T}]}{\partial a}<0,
$$

with the hypothesis that the initial open gate is chosen randomly between the two possible ones. 
If the first open gate is $\partial \Omega_{1}$ (i.e., if $N_{0}=1$ ), then the sign of $\partial_{a} \mathbb{E}_{1, x}[\mathcal{T}]$ is not guaranteed by the estimates of this paper for all $\boldsymbol{x} \in \Omega$. However, one can show that the quantity $(1 /|\Omega|) \int_{\Omega} \mathbb{E}_{1, \boldsymbol{x}}[\mathcal{T}] d \boldsymbol{x}$, which may be regarded as the MET, actually decays in $a$. In fact, we have from (3.7)

$$
\begin{aligned}
\frac{1}{|\Omega|} \int_{\Omega} \mathbb{E}_{1, \boldsymbol{x}}[\mathcal{T}] d \boldsymbol{x}= & \frac{1}{|\Omega|} \int_{\Omega} g(\boldsymbol{x}) d \boldsymbol{x}+\frac{C}{2}+\frac{A_{1}}{2} \frac{1}{|\Omega|} \int_{\Omega}\left(N_{\partial \Omega}+M_{\partial \Omega}\right)\left(\boldsymbol{x}, \boldsymbol{x}_{1}^{*}\right) d \boldsymbol{x} \\
& +\frac{A_{2}}{2} \frac{1}{|\Omega|} \int_{\Omega}\left(N_{\partial \Omega}-M_{\partial \Omega}\right)\left(\boldsymbol{x}, \boldsymbol{x}_{2}^{*}\right) d \boldsymbol{x},
\end{aligned}
$$

and hence

$$
\begin{aligned}
\frac{\partial}{\partial a}\left(\frac{1}{|\Omega|} \int_{\Omega} \mathbb{E}_{1, \boldsymbol{x}}[\mathcal{T}] d \boldsymbol{x}\right)= & \frac{1}{2|\Omega|} \frac{\partial C}{\partial a}+\frac{A_{1}}{2} \frac{\partial}{\partial a}\left(\frac{1}{|\Omega|} \int_{\Omega} R_{a}\left(\boldsymbol{x}, \boldsymbol{x}_{1}^{*}\right) d \boldsymbol{x}\right) \\
& -\frac{A_{2}}{2} \frac{\partial}{\partial a}\left(\frac{1}{|\Omega|} \int_{\Omega} R_{a}\left(\boldsymbol{x}, \boldsymbol{x}_{2}^{*}\right) d \boldsymbol{x}\right) .
\end{aligned}
$$

Thanks to (3.4), we have

$$
\frac{\partial}{\partial a} \int R_{a}\left(\boldsymbol{x}, \boldsymbol{x}_{j}^{*}\right) d \boldsymbol{x}=-\frac{1}{2 a^{2}}
$$

Thus we have

$$
\frac{\partial}{\partial a}\left(\frac{1}{|\Omega|} \int_{\Omega} \mathbb{E}_{1, \boldsymbol{x}}[\mathcal{T}] d \boldsymbol{x}\right)=\frac{1}{2|\Omega|} \frac{\partial C}{\partial a}+\frac{1}{2 a^{2}} \frac{\ln \frac{2}{\varepsilon_{2}}-\ln \frac{2}{\varepsilon_{1}}}{\ln \frac{2}{\varepsilon_{1}}+\ln \frac{2}{\varepsilon_{2}}}
$$

up to the term of order $O\left(\left(\ln \frac{2}{\varepsilon_{1}}+\ln \frac{2}{\varepsilon_{2}}\right)^{-1}\right)$, and therefore, if $\varepsilon_{1}=\varepsilon_{2}$, then the quantity $(1 /|\Omega|) \int_{\Omega} \mathbb{E}_{1, \boldsymbol{x}}[\mathcal{T}] d \boldsymbol{x}$ is decreasing in $a$. A similar reasoning shows the same for $(1 /|\Omega|) \int_{\Omega} \mathbb{E}_{2, \boldsymbol{x}}[\mathcal{T}] d \boldsymbol{x}$.

\section{Generalization to an arbitrary number of gates.}

6.1. The Markovian dynamics of the gates. Suppose that the boundary $\partial \Omega$ is partitioned into $n+1$ parts, $\partial \Omega_{1}, \ldots, \partial \Omega_{n}$, and the complementary $\partial \Omega_{c}$. We model an escape problem in which there are $n$ gates at the $\operatorname{arcs} \partial \Omega_{j}, j=1, \ldots, n$, that open alternatively: at time $t$ the open gate has index $N_{t}$ and $\left(N_{t}\right)_{t \geq 0}$ is a jump Markov process that is stepwise constant and takes values in $\{1, \ldots, n\}$. The Markovian dynamics can be described as follows: if at time $t$ the process is in state $k \in\{1, \ldots, n\}$, then during the small time interval $[t, t+h]$ the process can either jump to a new state or stay in $k$. The probability that it jumps to state $j \in\{1, \ldots, n\} \backslash\{k\}$ is:

$$
\mathbb{P}\left(N_{t+h}=j \mid N_{t}=k\right)=Q_{j k} h+o(h),
$$

and the probability that it stays in $k$ is

$$
\mathbb{P}\left(N_{t+h}=k \mid N_{t}=k\right)=1+Q_{k k} h+o(h) .
$$

We have $Q_{j k} \geq 0$ for $j \neq k, Q_{k k} \leq 0$, and $\sum_{j=1}^{n} Q_{j k}=0$. The $n \times n$ matrix $\mathbf{Q}=\left(Q_{j k}\right)_{j, k=1}^{n}$ characterizes the distribution of the Markov process $\left(N_{t}\right)_{t \geq 0}$ and it is called the infinitesimal generator. The transition probabilities $P(t, j \mid s, k)=\mathbb{P}\left(N_{t}=\right.$ $\left.j \mid N_{s}=k\right)$ satisfy the master equations:

$$
\frac{\partial P(t, j \mid s, k)}{\partial t}=\sum_{l=1}^{n} Q_{j l} P(t, l \mid s, k), \quad t \geq s, \quad P(s, j \mid s, k)=\delta_{j k}, \quad j, k=1, \ldots, n .
$$


Example 1. The telegraph process addressed in section 2 is a particular example with $n=2$ and

$$
\mathbf{Q}=a\left(\begin{array}{cc}
-1 & 1 \\
1 & -1
\end{array}\right)
$$

Example 2. Here $n \geq 2$. Assume that the Markov process is stepwise constant during time intervals whose durations follow independent and identically distributed exponential random variables with mean $1 / a$ and that the Markov process chooses another gate with equiprobability amongst the $n-1$ available gates when it jumps. Then

$$
\mathbf{Q}=\frac{a}{n-1}\left(\begin{array}{ccccc}
1-n & 1 & \ldots & \ldots & 1 \\
1 & 1-n & 1 & \ldots & 1 \\
\vdots & & \ddots & & \vdots \\
1 & \ldots & & 1-n & 1 \\
1 & \ldots & & 1 & 1-n
\end{array}\right)
$$

Example 3. Here $n \geq 3$. Assume that the Markov process is stepwise constant during time intervals whose durations follow independent and identically distributed exponential random variables with mean $1 / a$ and that the Markov process chooses either the right or the left gate with equiprobability when it jumps. Then

$$
\mathbf{Q}=\frac{a}{2}\left(\begin{array}{ccccccc}
-2 & 1 & 0 & \ldots & \ldots & 0 & 1 \\
1 & -2 & 1 & 0 & \ldots & \ldots & 0 \\
0 & 1 & -2 & 1 & 0 & \ldots & 0 \\
\vdots & & \ddots & \ddots & \ddots & & \vdots \\
0 & \ldots & 0 & 1 & -2 & 1 & 0 \\
0 & \ldots & \ldots & 0 & 1 & -2 & 1 \\
1 & 0 & \ldots & \ldots & 0 & 1 & -2
\end{array}\right)
$$

We assume that the Markov process is irreducible, i.e., there is a non-zero probability of transitioning (even if in more than one step) from any state to any other state. It means that for any $j \neq k, Q_{j k}>0$ or there exists $j_{1}, \ldots, j_{p}$ such that $Q_{j j_{1}} Q_{j_{1} j_{2}} \cdots Q_{j_{p} k}>0$.

We also assume that the process is reversible, i.e., the matrix $\mathbf{Q}$ is symmetric. Note that the process is irreducible and reversible in the three examples listed above.

The matrix $\mathbf{Q}$ is therefore diagonalizable:

$$
\mathbf{Q}=\mathbf{P D P}^{T}
$$

and, by Perron-Frobenius theorem, the matrix $\mathbf{Q}$ has one zero eigenvalue and the other eigenvalues are negative and denoted by

$$
\left(D_{11}, \ldots, D_{n n}\right)=\left(-a_{1},-a_{2}, \ldots,-a_{n}\right)
$$

with $a_{1}=0$ and $a_{j}>0, j \geq 2$. The eigenvector associated with the zero eigenvalue is the normalized uniform vector:

$$
P_{j 1}=\frac{1}{\sqrt{n}}, \quad j=1, \ldots, n .
$$


The process $\left(N_{t}\right)_{t \geq 0}$ is therefore ergodic and the transition probabilities $p(t, j \mid s, k)$ converge as $t \rightarrow \infty$ to the unique stationary distribution of the process which is the uniform distribution over $\{1, \ldots, n\}$.

The goal is to compute the expectation of the hitting time by the process $\left(\boldsymbol{X}_{t}\right)_{t \geq 0}$ of the time-dependent domain $\partial \Omega_{N_{t}}$. We denote by $\mathcal{T}$ the stopping time which corresponds to the "escape" of the particle:

$$
\mathcal{T}=\wedge_{j=1}^{n} \mathcal{T}_{j}, \quad \mathcal{T}_{j}=\inf \left\{t \geq 0, N_{t}=j \text { and } \boldsymbol{X}_{t} \in \partial \Omega_{j}\right\}, \quad j=1, \ldots, n .
$$

The MET is the expectation $\mathbb{E}_{k, x}[\mathcal{T}]$ of the stopping time when the initial states are $N_{0}=k$ and $\boldsymbol{X}_{0}=\boldsymbol{x}$.

Let us introduce the vector of functions $\boldsymbol{u}(\boldsymbol{x})=\left(u_{j}(\boldsymbol{x})\right)_{j=1, \ldots, n}^{T}$ which is the solution of the coupled linear system

$$
-\boldsymbol{j}=\mathbf{Q} \boldsymbol{u}(\boldsymbol{x})+\Delta \boldsymbol{u}(\boldsymbol{x}),
$$

where $\boldsymbol{j}=(1, \ldots, 1)^{T}$ and with the boundary conditions

$$
\begin{aligned}
& \left.\partial_{\nu} u_{j}\right|_{\partial \Omega_{c}}=0, \quad j=1, \ldots, n, \\
& \left.u_{j}\right|_{\partial \Omega_{j}}=0, \quad j=1, \ldots, n, \\
& \left.\partial_{\nu} u_{j}\right|_{\partial \Omega_{k}}=0, \quad j \neq k .
\end{aligned}
$$

Proceeding as in Section 2, we have the following proposition for the MET:

Proposition 6.1. For $\boldsymbol{x} \in \Omega$ and $k=1, \ldots, n$,

$$
\mathbb{E}_{k, \boldsymbol{x}}[\mathcal{T}]=u_{k}(\boldsymbol{x})
$$

6.2. Integral equations. We introduce $\boldsymbol{v}=\mathbf{P}^{T} \boldsymbol{u}$. For $k=1, \ldots, n$ the function $v_{k}=\sum_{j=1}^{n} P_{j k} u_{j}, k=1, \ldots, n$, satisfies

$$
\triangle v_{k}-a_{k} v_{k}=-\sum_{j=1}^{n} P_{j k} .
$$

Using (6.3) and the orthogonality of the eigenvectors of $\mathbf{Q}$ we have

$$
\sum_{j=1}^{n} P_{j k}= \begin{cases}\sqrt{n} & \text { if } k=1 \\ 0 & \text { if } k \geq 2\end{cases}
$$

Therefore the Green's formula gives us

$$
v_{k}(\boldsymbol{z})=\sqrt{n} \delta_{k 1} g(\boldsymbol{z})+\delta_{k 1} \frac{1}{\partial \Omega \mid} \int_{\partial \Omega} v_{1}(\boldsymbol{x}) d \sigma(\boldsymbol{x})+\int_{\partial \Omega} M^{a_{k} / 2}(\boldsymbol{x}, \boldsymbol{z}) \partial_{\nu} v_{k}(\boldsymbol{x}) d \sigma(\boldsymbol{x}),
$$

for $\boldsymbol{z} \in \Omega$. Here we have set $M^{0}=N$, the Neumann function. Since $\boldsymbol{u}=\mathbf{P} \boldsymbol{v}$, we can show that the following representation hold for all $l=1, \ldots, n$ and for all $\boldsymbol{z} \in \Omega$ :

$$
\begin{aligned}
u_{l}(\boldsymbol{z})= & g(\boldsymbol{z})+\frac{C}{n}+\sum_{j=1}^{n} \int_{\partial \Omega_{j}} \partial_{\nu} u_{j}(\boldsymbol{x}) \sum_{k=1}^{n} P_{l k} P_{j k} M^{a_{k} / 2}(\boldsymbol{x}, \boldsymbol{z}) d \sigma(\boldsymbol{x}) \\
= & g(\boldsymbol{z})+\frac{C}{n}-\frac{1}{\pi} \int_{\partial \Omega_{l}} \ln |\boldsymbol{x}-\boldsymbol{z}| \partial_{\nu} u_{l}(\boldsymbol{x}) d \sigma(\boldsymbol{x}) \\
& +\sum_{j=1}^{n} \int_{\partial \Omega_{j}} \partial_{\nu} u_{j}(\boldsymbol{x}) \sum_{k=1}^{n} P_{l k} P_{j k} S_{k}(\boldsymbol{x}, \boldsymbol{z}) d \sigma(\boldsymbol{x}),
\end{aligned}
$$


where $C$ is the constant defined by

$$
C=\frac{1}{|\partial \Omega|} \sum_{j=1}^{n} \int_{\partial \Omega} u_{j}(\boldsymbol{x}) d \sigma(\boldsymbol{x})
$$

and

$$
S_{1}(\boldsymbol{x}, \boldsymbol{z})=R(\boldsymbol{x}, \boldsymbol{z}), \quad S_{k}(\boldsymbol{x}, \boldsymbol{z})=R_{a_{k} / 2}(\boldsymbol{x}, \boldsymbol{z}), \quad k=2, \ldots, n .
$$

Here $R$ and $R_{a}$ are functions defined by (3.2) and (3.5), respectively. We then find that for all $l=1, \ldots, n$ and for all $z \in \partial \Omega_{l}$ :

$$
\begin{aligned}
& g(\boldsymbol{z})+\frac{C}{n}-\frac{1}{\pi} \int_{\partial \Omega_{l}} \ln |\boldsymbol{x}-\boldsymbol{z}| \partial_{\nu} u_{l}(\boldsymbol{x}) d \sigma(\boldsymbol{x}) \\
& +\sum_{j=1}^{n} \int_{\partial \Omega_{j}} \partial_{\nu} u_{j}(\boldsymbol{x}) \sum_{k=1}^{n} P_{l k} P_{j k} S_{k}(\boldsymbol{x}, \boldsymbol{z}) d \sigma(\boldsymbol{x})=0 .
\end{aligned}
$$

6.3. Asymptotic expansions. Denoting by $\boldsymbol{x}_{j}^{*}$ the centers of the arc $\partial \Omega_{j}$ and by $\varepsilon_{j} \ll 1$ its half-length, we find that the numbers $A_{j}$ defined by

$$
A_{j}=\int_{\partial \Omega_{j}} \partial_{\nu} u_{j}(\boldsymbol{x}) d \sigma(\boldsymbol{x}), \quad j=1, \ldots, n,
$$

satisfy the $n$ equations

$$
g\left(\boldsymbol{x}_{l}^{*}\right)+\frac{C}{n}-\frac{\ln \varepsilon_{l} / 2}{\pi} A_{l}+\sum_{j=1}^{n}\left(\sum_{k=1}^{n} P_{l k} P_{j k} S_{k}\left(\boldsymbol{x}_{j}^{*}, \boldsymbol{x}_{l}^{*}\right)\right) A_{j}=O(\varepsilon), \quad l=1, \ldots, n,
$$

together with the compatibility condition

$$
\sum_{j=1}^{n} A_{j}=-n|\Omega|
$$

Therefore, the $n+1$ linear equations (6.14) and (6.15) determine the constant $C$ and $A_{j}, j=1, \ldots, n$ (up to terms of order $\varepsilon$ ) and it can then be substituted in (6.9) to get the expansion of the MET:

$$
u_{l}(\boldsymbol{z})=g(\boldsymbol{z})+\frac{C}{n}+\sum_{j=1}^{n} \sum_{k=1}^{n} A_{j} P_{l k} P_{j k} M^{a_{k} / 2}\left(\boldsymbol{x}_{j}^{*}, \boldsymbol{z}\right)+O(\epsilon)
$$

for $l=1, \ldots, n$ and $\boldsymbol{z} \in \Omega$ away from $\boldsymbol{x}_{j}^{*}, j=1, \ldots, n$.

To leading order in $\varepsilon$ we find that

$$
\frac{C}{n} \simeq \frac{|\Omega|}{\pi}\left(\frac{1}{n} \sum_{l=1}^{n} \frac{1}{\ln \frac{2}{\varepsilon_{l}}}\right)^{-1}+O(1)
$$

and one can see that the leading-order term of the MET is independent of the initial state $N_{0}$ and of the detailed dynamics of the Markov process:

$$
\mathbb{E}_{k, \boldsymbol{x}}[\mathcal{T}]=\frac{|\Omega|}{\pi}\left(\frac{1}{n} \sum_{l=1}^{n} \frac{1}{\ln \frac{2}{\varepsilon_{l}}}\right)^{-1}+O(1)
$$


7. Conclusion. In this paper we have considered the narrow escape problem and derived the asymptotic expansion of the mean escape time when there are multiple gates which open alternatively according to Markovian dynamics. The major findings of this paper are (i) The leading order term of the asymptotic of the MET does not depend on the initial state or the particular dynamics of the Markov process; it only depends of the number of gates and their sizes, (ii) when there are two switching gates, the leading order term of the MET is twice that when there are two gates which open all the time, (iii) the higher the switching rate is, the shorter is the MET in its first order correction. We finally remark that it would be interesting to extend the result of this paper to three dimensions.

\section{REFERENCES}

[1] H. Ammari and H. Kang, Polarization and Moment Tensors with Applications to Inverse Problems and Effective Medium Theory, Applied Mathematical Sciences, Vol. 162, SpringerVerlag, New York, 2007.

[2] H. Ammari, H. Kang, and H. Lee, Layer Potential Techniques in Spectral Analysis, Mathematical Surveys and Monographs, Vol. 153, Amer. Math. Soc., Providence RI, 2009.

[3] H. Ammari, H. Kang, K. Kalimeris, and H. Lee, Layer potential techniques for the narrow escape problem, submitted.

[4] J.B. Bardet, H. Guérin, and F. Malrieu, Long time behavior of diffusions with Markov switching, Alea 7 (2010), 151-170.

[5] A.F. Cheviakov, M.J. Ward, and R. Straube, An asymptotic analysis of the mean first passage time for narrow escape problems: Part II: The sphere, preprint.

[6] S. Goldstein, On diffusion by discontinuous movements and the telegraph equation, The Quarterly Journal of Mechanics and Applied Mathematics, 4 (1951), 129-156.

[7] D. Holcman, Diffusion in cellular microdomains: application to synapses, Contemp. Math., Vol. 494, (ed. H. Ammari and H. Kang), 1-26, Amer. Math. Soc., Providence RI, 2009.

[8] D. Holcman and Z. Schuss, Escape through a small opening: receptor trafficking in a synaptic membrane, J. Stat. Phys., 117 (2004), 975-1014.

[9] D. Holcman and Z. Schuss, Diffusion escape through a cluster of small absorbing windows, J. Phys. A: Math. Theor., 41 (2008), 155001.

[10] D. Holcman and Z. Schuss, Diffusion through a cluster of small windows and flux regulation in microdomains, Phys. Lett. A, 372 (2008), 3768-3772.

[11] P. L. Lions and A. S. Sznitman, Stochastic differential equations with reflecting boundary conditions. Comm. Pure Appl. Math., 37 (1984), 511-537.

[12] S. Pillay, M.J. Ward, A. Peirce, and T. Kolokolnikov, An asymptotic analysis of the mean first passage time for the narrow escape problems: Part I: two-dimensional domain, preprint.

[13] J. Reingruber and D. Holcman, Gated narrow escape time for molecular signaling, Phys. Rev. Lett. 103, 148102 (2009).

[14] Z. Schuss, A. Singer, and D. Holcman, The narrow escape problem for diffusion in cellular microdomains, Proc. Nat. Acad. Sci., 104 (2007), 16098-16103.

[15] A. Singer and Z. Schuss, Activation through a narrow opening, SIAM J. Appl. Math., 68 (2007), 98-108.

[16] A. Singer, Z. Schuss, and D. Holcman, Narrow escape and leakage of Brownian particles, Phys. Rev. E, 78 (2008), 051111.

[17] A. Singer, Z. Schuss, D. Holcman, and R.S. Eisenberg, Narrow escape. I., J. Stat. Phys., 122 (2006), 437-463.

[18] A. Singer, Z. Schuss, and D. Holcman, Narrow escape, Part II: The circular disk, J. Stat. Phys., 122 (2006), 465-489.

[19] A. Singer, Z. Schuss, and D. Holcman, Narrow escape. III. Non-smooth domains and Riemann surfaces, J. Stat. Phys. 122 (2006), 491-509. 